\title{
Individualized Embryo Selection Strategy Developed by Stacking Machine Learning Model for Better In Vitro Fertilization Outcomes: An Application Study.
}

\section{Qingsong XI}

Tongji Hospital of Tongji Medical College of Huazhong University of Science and Technology Qiyu YANG

Tongji Hospital of Tongji Medical College of Huazhong University of Science and Technology Meng WANG

Tongji Hospital of Tongji Medical College of Huazhong University of Science and Technology

\section{Bo HUANG}

Tongji Hospital of Tongji Medical College of Huazhong University of Science and Technology

\section{Bo ZHANG}

Tongji Hospital of Tongji Medical College of Huazhong University of Science and Technology

\section{Zhou LI}

Tongji Hospital of Tongji Medical College of Huazhong University of Science and Technology

\section{Shuai LIU}

Tongji Hospital of Tongji Medical College of Huazhong University of Science and Technology

\section{Liu YANG}

Tongji Hospital of Tongji Medical College of Huazhong University of Science and Technology

\section{Lixia ZHU}

Tongji Hospital of Tongji Medical College of Huazhong University of Science and Technology

Lei Jin ( $\sim$ leijintongjih@qq.com )

Tongji Hospital of Tongji Medical College of Huazhong University of Science and Technology https://orcid.org/0000-0003-0367-7765

\section{Research}

Keywords: Artificial intelligence, embryo selection, machine learning, in vitro fertilization, in vitro fertilization prediction

Posted Date: January 11th, 2021

DOI: https://doi.org/10.21203/rs.3.rs-140383/v1 
License: (c) (i) This work is licensed under a Creative Commons Attribution 4.0 International License. Read Full License

Version of Record: A version of this preprint was published at Reproductive Biology and Endocrinology on April 5th, 2021. See the published version at https://doi.org/10.1186/s12958-021-00734-z. 


\section{Abstract}

Background: To minimize the rate of in vitro fertilization (IVF)- associated multiple-embryo gestation, significant efforts have been made. Previous studies related to machine learning in IVF mainly focused on selecting the top-quality embryos to improve outcomes, however, in patients with sub-optimal prognosis or with medium- or inferior-quality embryos, the selection between SET and DET could be perplexing.

Methods: This was an application study including 7887 patients with 8585 embryos treated during 2016 to 2018, in Tongji Hospital, Wuhan, China. A hierarchical model was established using the machine learning system XGBoost, to learn embryo implantation potential and the impact of double embryos transfer (DET) simultaneously. The performance of the model was evaluated with the AUC of the ROC curve. Multiple regression analyses were also conducted on the 19 selected features to demonstrate the differences between feature importance for prediction and statistical relationship with outcomes.

Results: For a single embryo transfer (SET) pregnancy, the following variables remained significant: age, attempts at IVF, estradiol level on hCG day, and endometrial thickness. For DET pregnancy, age, attempts at IVF, endometrial thickness, and the newly added P1+P2 remained significant. For DET twin risk, age, attempts at IVF, 2PN/ MII, and P1×P2 remained significant. The algorithm was repeated 30 times, and averaged AUC of $0.7945,0.8385$, and 0.7229 were achieved for SET pregnancy, DET pregnancy, and DET twin risk, respectively. The trend of predictive and observed rates both in pregnancy and twin risk was basically identical. XGBoost outperformed the other two algorithms: logistic regression and classification and regression tree.

Conclusion: Artificial intelligence based on determinant-weighting analysis could offer an individualized embryo selection strategy for any given patient, and predict clinical pregnancy rate and twin risk, therefore optimizing clinical outcomes.

\section{Introduction}

For decades, discussions about how to improve the clinical outcomes of in vitro fertilization (IVF) treatment have persisted. Multiple-embryo transfer was suggested to increase the possibility of successful implantation but inevitably elevated the risk of multiple-embryo gestation. IVF-associated multiple pregnancies exhibit significant financial, social, and medical implications [1, 2]. Even though the transfer of embryos has been limited to no more than two in recent years, the overall twin rate worldwide after assisted reproduction has still varied from 15-30\% [3]. The incidences of premature birth, low birth weight, cerebral palsy, neurological complications, and perinatal mortality of twin pregnancy markedly increased compared with singleton pregnancy [4]. To minimize the rate of multiple-embryo gestation, significant efforts, including individualized service provision and single embryo transfer (SET) enhancement, have been made in the course of these decades. 
Embryo morphological analysis is the routine method for selecting the highest-quality embryos to transfer. It is commonly suggested to perform SET on prognostically good patients with a top-quality embryo and to perform double embryo transfer (DET) in prognostically poor patients because no goodquality embryos are available in the IVF lab. In a large proportion of IVF patients with sub-optimal prognosis or with medium- or inferior-quality embryos, the selection between SET and DET could be perplexing. Because many features have been shown to influence embryo implantation potential positively or negatively in IVF [5-8], developing an optimal embryo selection plan to balance maximum clinical pregnancy rate and minimum twin risk is complicated for IVF clinicians. Therefore, a more precise, quantified, stable embryo selection model based on all possible influencing determinants needs to be constructed. This will potentially offer evidence-based patient counseling and predictable successful chances for any given patient.

Artificial intelligence (Al) represents the combination of machine learning, and a moderation and selfadapting prediction model. Previous studies related to machine learning in IVF mainly focused on selecting the top-quality embryos to improve IVF outcomes [9-13]. Developing a flexible, individualized embryo selection approach based on available embryos of various qualities and different twin rate threshold settings is another promising issue.

The purpose of our study is to construct an individualized embryo selection strategy and pregnancy prediction model, developed by stacking machine learning, to identify features correlated with embryo implantation potential and to evaluate available embryos' implantation chances quantitatively. We aim to balance maximal clinical pregnancy and minimal acceptable twin risk in IVF with this model and validate its clinical effectiveness and practicability in subsequent cycles..

\section{Materials And Methods}

\section{Study design and participants}

IVF patients in Tongji Hospital between January 2016 and December 2018, with one or two embryo transfers in the fresh cycle, were enrolled in our study. Exclusion criteria included patients with (1) blastocyst transfer in fresh cycle; (2) oocyte donation cycles; (3) vitrified/warmed oocytes; (4) oocytes partially cryopreserved in fresh cycle; (5) combined vitrified/warmed embryo transfers. In total, 4926 patients, between January 2016 and March 2018 were included as the training set to construct our model. Thirty-eight features were analyzed in our study, and their baselines on training and validation sets were exhibited, among which 21 main variables were selected and listed in Table 1, including patient features, embryo morphology features, and embryo scores. After model construction, the developed embryo selection strategy was applied to guide the selection of SET or DET in 2961 individual cases between April and December 2018 as the validation set. 
Table 1

Baseline characteristics of the variables included in the training and validation data sets.

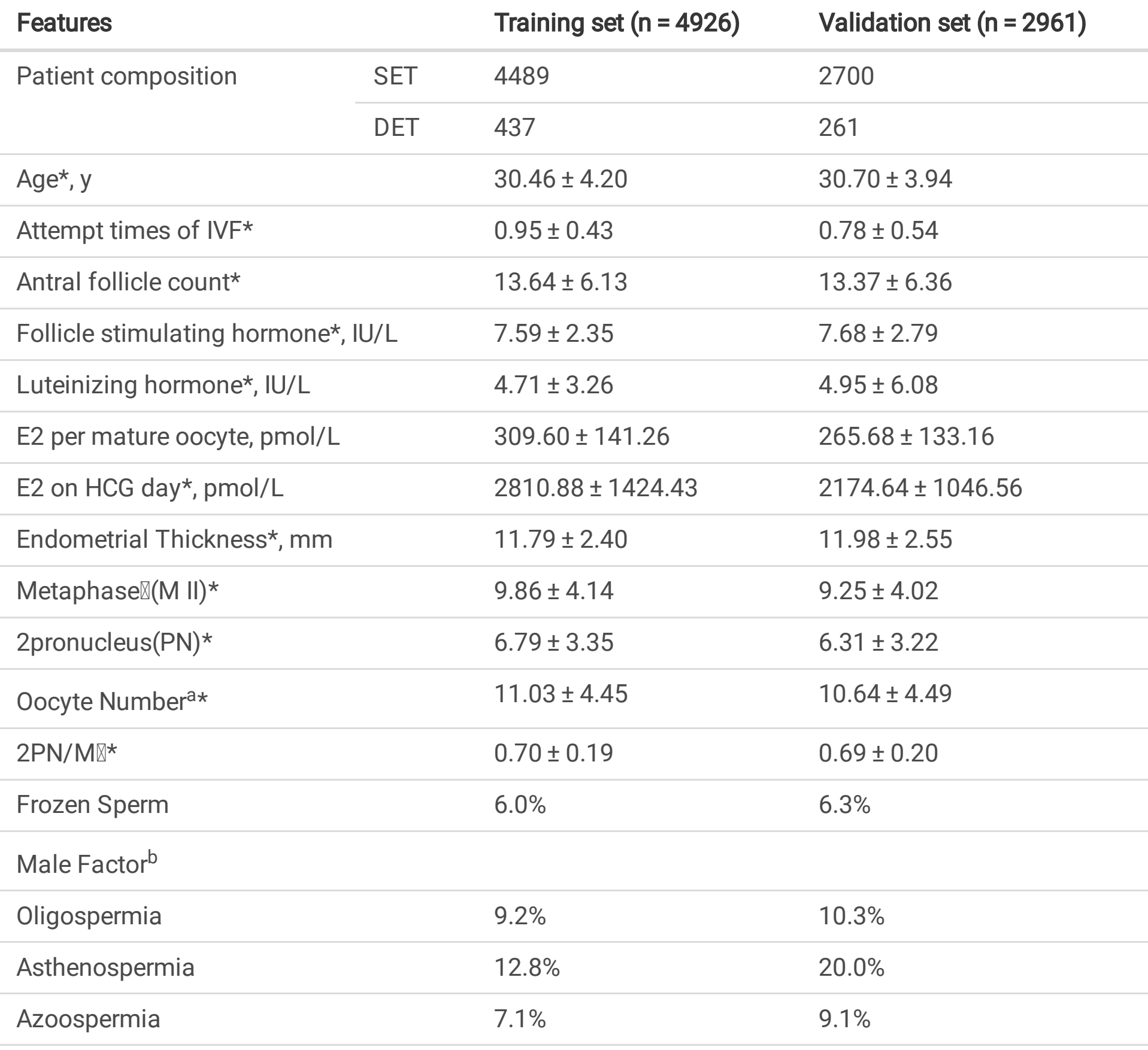

*The selected features after performing feature selection are marked by asterisks.

SET, single-embryo transfer; DET, double-embryo transfer; IVF, in vitro fertilization; ICSI, intracytoplasmic sperm injection; E2: estradiol; hCG, human chorionic gonadotropin; MESA, microscopic epididymal sperm aspiration; TESA, testicular sperm aspiration; PESA, percutaneous epididymal sperm aspiration.

(a) Number of oocytes retrieved; (b) for multi-category features, the sum of the proportion for each category may not equal $100 \%$ because the missing value exists or another small proportion of category features is not included; (c) infertility is encoded by 0 or 1 if the patient is primary or secondary, respectively; (d) the fragment is encoded by three values: 1 to 3 representing no fragment, $5 \%-15 \%$ fragment, and $>15 \%$ fragment, respectively; (e) the equality is encoded by five values, 0 to 4 , and represent equal, sort of equal, unequal, sort very unequal, and very unequal, respectively. 


\section{Features}

Female Factor ${ }^{b}$

Endometriosis

Ovulation Disorder

Unknown

Sperm Retrieval

Ejaculation

$95.2 \%$

$95.2 \%$

MESA

$0.3 \%$

$0.7 \%$

TESA

$1.0 \%$

$3.5 \%$

PESA

Stimulation Protocol ${ }^{b}$

Agonist Protocol*

Antagonist Protocol

$71.4 \%$

$22.8 \%$

$70.8 \%$

Endometrial Type ${ }^{\mathrm{b}}$

$A^{*}$

$83.3 \%$

$2.0 \%$

$21.4 \%$

$C^{*}$

Infertility ${ }^{c}$

Primary

Secondary*

$64.9 \%$

$70.4 \%$

$35.1 \%$

$29.6 \%$

Fertilization Method ${ }^{\mathrm{b}}$

*The selected features after performing feature selection are marked by asterisks.

SET, single-embryo transfer; DET, double-embryo transfer; IVF, in vitro fertilization; ICSI, intracytoplasmic sperm injection; E2: estradiol; hCG, human chorionic gonadotropin; MESA, microscopic epididymal sperm aspiration; TESA, testicular sperm aspiration; PESA, percutaneous epididymal sperm aspiration.

(a) Number of oocytes retrieved; (b) for multi-category features, the sum of the proportion for each category may not equal $100 \%$ because the missing value exists or another small proportion of category features is not included; (c) infertility is encoded by 0 or 1 if the patient is primary or secondary, respectively; (d) the fragment is encoded by three values: 1 to 3 representing no fragment, $5 \%-15 \%$ fragment, and $>15 \%$ fragment, respectively; (e) the equality is encoded by five values, 0 to 4 , and represent equal, sort of equal, unequal, sort very unequal, and very unequal, respectively. 


\begin{tabular}{|c|c|c|}
\hline Features & Training set $(n=4926)$ & Validation set $(n=2961)$ \\
\hline IVF & $72.5 \%$ & $57.9 \%$ \\
\hline $\mid C S I^{*}$ & $24.5 \%$ & $34.8 \%$ \\
\hline \multicolumn{3}{|l|}{ Embryo Features } \\
\hline Number of Blastomere* & $7.93 \pm 0.89$ & $8.06 \pm 0.93$ \\
\hline Fragment ${ }^{\mathrm{d} *}$ & $0.37 \pm 0.50$ & $0.32 \pm 0.48$ \\
\hline Equality ${ }^{\mathrm{e} *}$ & $0.91 \pm 0.95$ & $0.87 \pm 0.91$ \\
\hline \multicolumn{3}{|c|}{ *The selected features after performing feature selection are marked by asterisks. } \\
\hline \multicolumn{3}{|c|}{$\begin{array}{l}\text { SET, single-embryo transfer; DET, double-embryo transfer; IVF, in vitro fertilization; ICSI, } \\
\text { intracytoplasmic sperm injection; E2: estradiol; hCG, human chorionic gonadotropin; MESA, } \\
\text { microscopic epididymal sperm aspiration; TESA, testicular sperm aspiration; PESA, percutaneous } \\
\text { epididymal sperm aspiration. }\end{array}$} \\
\hline \multicolumn{3}{|c|}{$\begin{array}{l}\text { (a) Number of oocytes retrieved; (b) for multi-category features, the sum of the proportion for each } \\
\text { category may not equal } 100 \% \text { because the missing value exists or another small proportion of } \\
\text { category features is not included; (c) infertility is encoded by } 0 \text { or } 1 \text { if the patient is primary or } \\
\text { secondary, respectively; (d) the fragment is encoded by three values: } 1 \text { to } 3 \text { representing no fragment, } \\
5 \%-15 \% \text { fragment, and }>15 \% \text { fragment, respectively; (e) the equality is encoded by five values, } 0 \text { to } 4 \text {, } \\
\text { and represent equal, sort of equal, unequal, sort very unequal, and very unequal, respectively. }\end{array}$} \\
\hline
\end{tabular}

\section{Controlled Ovarian Hyperstimulation, Embryo Culture, And Pregnancy Ascertain}

IVF patients were treated with controlled ovarian hyperstimulation $(\mathrm{COH})$ by gonadotropin-releasing hormone $(\mathrm{GnRH})$ agonist or $\mathrm{GnRH}$ antagonist, as previously described $[14,15]$. When two dominant follicles reached 17-18 $\mathrm{mm}$ in diameter, oocytes were retrieved transvaginally 36-38 hours after a human chorionic gonadotropin (hCG) trigger. Embryos were cultured in G1 medium (Vitrolife, Sweden) after fertilization and transferred on day 3. Subsequently, biochemical pregnancy was ascertained by a positive serum hCG two weeks after embryo transfer with serial elevation. Clinical pregnancy was defined as a gestational sac and active fetal heartbeat on ultrasound demonstration eight weeks after embryo transfer.

\section{Model Construction, Feature Selection, And Validation}

According to previous studies, the outcome of DET is not a simple binomial distribution with respect to two transferred embryos [16]. Therefore, a hierarchical model with two levels was established to learn single embryo implantation potential and the impact of double embryos transferred simultaneously. In the first level, patient and embryo features were applied to predict the implantation chance for single 
embryo, from which the output value was directly treated as the predicted pregnancy chance. For DET, another two features, namely $\mathrm{P} 1+\mathrm{P} 2$ and $\mathrm{P} 1 \times \mathrm{P} 2$, were generated, using the $\mathrm{P} 1$ and $\mathrm{P} 2$ values predicted by the first level for two embryos, followed by the combination of generated features and patient features as the input for second level. Two models were developed in the second level, one to predict the DET pregnancy chance and the other to predict the DET twin risk. The overall flowchart of our model is shown in Fig. 1. The first-level model was trained by all data except DET with only one embryo implantation, whereas the second-level models were trained by all DET data.

The machine learning system applied here was XGBoost [17]. XGBoost is a scalable machine learning system for tree boosting, which uses the boosting technique to train multiple trees. In other words, $X G B o o s t$ is an ensemble of multiple decision trees. Unlike random forest, which generates trees by randomly selecting a subset of training sets and features, XGBoost generates decision trees one by one, based on the performance of the previous generated trees. And comparing to single decision tree algorithm such as C4.5 or CART, ensemble of trees can achieve better performance. Moreover, this system can automatically deal with missing values and assign the importance score for each feature, which was applied for feature selection. Nineteen features were selected and are marked by asterisks in Table 1.

As the training procedure was completed, the performance of the model was evaluated with the area under the curve (AUC) of the receiver operating characteristic (ROC) curve. Multiple regression analyses were also conducted on the 19 selected features, with the glm function in $\mathrm{R}$ to demonstrate the differences between feature importance for prediction and statistical relationship with outcomes.

\section{Embryo Selection Strategy Development}

Subsequently, an embryo selection strategy to maximize the pregnancy chance with a controlled twin rate threshold setting was developed. As shown in Fig. 2, for any given acceptable twin rate threshold, the pregnancy and twin rate could be predicted for every possible embryo selection strategy, including one or two best embryos to transfer or the combination of one best embryo and one medium-quality embryo. Moreover, if the transfer failed in the fresh cycle, the plan would be redeveloped with the remaining embryos, following the same protocol until all embryos were transferred or a new cycle was started. With this selection strategy, the prediction model could effectively increase the percentages of elective SET patients and provide plausible plans for DET patients in IVF.

\section{Statistical analysis}

Continuous variables were presented as mean \pm standard deviation. Categorical variables were presented as percentage encoded by one-hot encoding for analysis. For XGBoost algorithm, we used the implementation from scikit-learn (https://xgboost.readthedocs.io/en/latest/python/ python_api.html) using Python. The parameters were as follows: $\max \_d e p t h=5$, min_child_weight $=1$, learning_rate $=0.1$, n_estimators $=100$, gamma $=0$, subsample $=0.8$, colsample_bytree $=0.8$. Other parameters were set by default. 


\section{Results}

\section{Variable analysis using training data (January 2016 to March 2018)}

The results of multiple regression analyses are shown in Table 2. For a SET pregnancy, the following variables remained significant: age, attempts at IVF, estradiol level on hCG day, and endometrial thickness. For DET pregnancy, age, attempts at IVF, endometrial thickness, and the newly added P1 + P2 remained significant. For DET twin risk, age, attempts at IVF, 2 pronucleus (PN)/ metaphase II (M II), and P1 × P2 remained significant. 
Table 2

Multivariate analysis results of the selected features for SET pregnancy, DET pregnancy and twin risk prediction.

\begin{tabular}{|c|c|c|c|}
\hline \multirow[t]{3}{*}{ Selected features } & \multicolumn{3}{|l|}{$P$ value } \\
\hline & \multirow{2}{*}{$\begin{array}{l}\text { SET } \\
\text { Pregnancy }\end{array}$} & \multicolumn{2}{|l|}{ DET } \\
\hline & & Pregnancy & Twin Risk \\
\hline Age & $0.0222^{*}$ & $<0.0001^{\star}$ & $<0.0001^{\star}$ \\
\hline Attempt times of IVF & $<0.001^{\star}$ & $<0.0001 *$ & $<0.0001^{*}$ \\
\hline Antral follicle count & 0.3332 & 0.4016 & 0.4121 \\
\hline Follicle stimulating hormone & 0.7307 & 0.8141 & 0.4633 \\
\hline Luteinizing hormone & 0.3501 & 0.7230 & 0.4616 \\
\hline E2 on HCG day & $0.0053^{*}$ & 0.9040 & 0.7684 \\
\hline Endometrial Thickness & $0.0046^{*}$ & $<0.0001^{\star}$ & 0.2080 \\
\hline $\mathrm{M} \mathbb{\mathrm { V }}$ & 0.9455 & 0.9444 & 0.0546 \\
\hline $2 \mathrm{PN}$ & 0.1041 & 0.9068 & 0.1021 \\
\hline Oocyte Number & 0.7510 & 0.8897 & 0.6324 \\
\hline 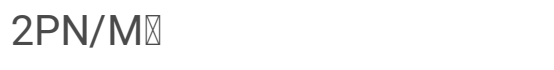 & 0.5038 & 0.7772 & $0.0148^{*}$ \\
\hline \multicolumn{4}{|l|}{ Stimulation Protocol } \\
\hline Agonist Protocol & 0.3692 & 0.2019 & 0.4961 \\
\hline \multicolumn{4}{|l|}{ Endometrial Type } \\
\hline A & 0.8531 & 0.5324 & 0.4914 \\
\hline C & 0.7138 & 0.0887 & 0.4614 \\
\hline Secondary Infertility & 0.0816 & 0.2445 & 0.6809 \\
\hline \multicolumn{4}{|l|}{ Fertilization Method } \\
\hline ICSI & 0.2365 & 0.5069 & 0.8492 \\
\hline \multicolumn{4}{|l|}{ Embryo Features } \\
\hline Blastomere Number & 0.5422 & $N U^{a}$ & $\mathrm{NU}$ \\
\hline Fragment & 0.1585 & NU & $\mathrm{NU}$ \\
\hline Equality & 0.5399 & NU & NU \\
\hline
\end{tabular}

${ }^{*} \mathrm{P}<0.05 ;{ }^{a} \mathrm{NU}$ means this feature was not used in the corresponding level. 


\begin{tabular}{|c|c|c|c|}
\hline \multirow[t]{3}{*}{ Selected features } & \multicolumn{3}{|l|}{$P$ value } \\
\hline & \multirow{2}{*}{$\begin{array}{l}\text { SET } \\
\text { Pregnancy }\end{array}$} & \multicolumn{2}{|l|}{ DET } \\
\hline & & Pregnancy & Twin Risk \\
\hline \multicolumn{4}{|l|}{ Embryo Scores } \\
\hline $\mathrm{P} 1+\mathrm{P} 2$ & NU & $<0.001^{\star}$ & 0.1344 \\
\hline $\mathrm{P} 1 \times \mathrm{P} 2$ & NU & 0.2040 & $0.007^{\star}$ \\
\hline
\end{tabular}

\section{Feature Importance Of The Hierarchical Model}

In the XGBoost algorithm, the feature importance means the number of times that a feature is used to split the data across all trees. A higher feature importance score represents greater value for prediction. Figure 3 shows the feature importance in the hierarchical model for a SET pregnancy, a DET pregnancy, and DET twin risk. Some features are not shown in Fig. 3 because the corresponding importance was zero, which means these features were not used for prediction.

For SET, as shown in Table 2, the luteinizing hormone (LH) level and follicle-stimulating hormone (FSH) level were not statistically significant, yet they were the third- and sixth-important features for the firstlevel model. The same phenomenon was observed for FSH, LH, and antral follicle count (AFC) in DET pregnancy and DET twin risk prediction. Although $\mathrm{P} 1 \times \mathrm{P} 2$ was significantly correlated with DET twin risk, it was hardly used by either level model.

\section{Model Validation Results}

The algorithm was repeated 30 times to eliminate the interference of random factors, and averaged AUCs of $0.7945,0.8385$, and 0.7229 were achieved for SET pregnancy, DET pregnancy, and DET twin risk, respectively. The ROC curve in one single run is shown in Fig. 4.

To validate the effectiveness of the developed embryo selection strategy, the consistency between the predicted chance and the observed actual outcomes was analyzed, as shown in Fig. 5. The trend of predictive and observed rates both in pregnancy and twin risk was basically identical, although with a slight elevation in predictive rates.

To validate the prediction performance among XGBoost and other algorithms such as logistic regression (LR) and classification and regression tree (CART), we also performed a non-parametric multiple comparison test using Dunn's procedure [18], with a p-value correction using the False Discovery Rate method [19]. The results are shown in Fig. 6 , where the significant ones $(p<0.05)$ are marked in asterisk. 
In general, XGBoost outperformed the other two algorithms with obvious prediction power in SET pregnancy prediction and DET twin risk prediction and similar performance in DET pregnancy prediction task.

\section{Discussion}

One of the most overwhelming challenges in contemporary assisted reproductive technology (ART) is how to narrow the gap in perinatal and neonatal outcomes between spontaneous pregnancy and assisted pregnancy, usually caused by multiple-embryo transfer and implantation [20]. In recent decades, SET has been advocated in IVF to prevent multiple pregnancies while led to the concerns of decreased IVF success $[3,21,22]$. Therefore, precise embryo selection for SET and twin risk warning for DET are particularly necessary. In this paper, a novel hierarchical model was constructed and validated to optimize embryo selection strategies and successfully predict pregnancy for both SET and DET, as well as predict the twin risk of DET for each individual. Subsequently, we validated our model on 2961 patients, and the results showed that our model had achieved an acceptable performance on embryo selection and twin risk prediction for each individual, as shown in Fig. 4 and Fig. 5.

It is generally accepted that embryos with similar morphology present variable implantation probability, depending on other assessed features such as patient characteristics and cycle demographics. Many features, including age, FSH level, anti-Mullerian hormone (AMH) and embryo quality, have been reported as independent impact factors on oocyte variability and embryo implantation potential [5, 23-26]. Machine learning is considered a powerful mathematical tool for correlation analysis when huge data is involved; therefore, Al has been introduced in embryo variability prediction in many articles $[27,28]$. By increasing the information of the input features, the predictive power of the proposed model may be improved. Similarly, 19 features from the initial data sets were considered in the current study to construct the predictive power in our model. It is noteworthy that although previous models may have helped predict the implantation potential of a specific embryo, they did not offer a reasonable and optimal guidance to the embryo selection determination in clinical practice.

Minaretzis et al. [29] once presented an embryo selection strategy of transferring one additional goodquality embryo for each five years of incremental increase in maternal age to improve IVF outcome by multivariate analysis of factors predictive of successful live birth. Because maternal age was the only influencing factor to be considered, the recommended strategy was not so accurate or comprehensive. Kaufmann et al. [30] reported a neural networks predicting model in IVF, where four input parameters were included and the overall accuracy was 59\%. Uyar et al. [31] proposed a Support Vector Machine (SVM) method in embryo implantation prediction in terms of Area Under ROC curve (0.712 \pm 0.032$)$, when 12 features were included.

Vaegter et al. [16] also constructed a predictive model for an embryo transfer strategy, in which only one embryo was transferred if the predictive risk of twin implantation was above 15\%, using the two highest scored embryos. While validating this predictive model, the actual twin rate was $3.8 \%$, which was far 
lower than the expected 15\% setting, accompanied by a decreased live birth rate. Unlike previous studies, a novel strategy in embryo selection based on an accurate embryo potential prediction and twin risk assessment was developed in our model. The model not only guides a determination of the number of embryos for transfer and the specific embryo selection, but also presents a relatively accurate prediction of the pregnancy rate and twin risk of the corresponding selection scheme.

Because SET was not arbitrarily implemented in all IVF patients, twin pregnancy was inevitable. However, the acceptable twin rate threshold varied among different countries, even among different IVF centers. The greatest strength of our model was that the embryo selection strategy we developed varied correspondingly to patients' characteristics and embryo morphology parameters as long as different twin rate thresholds were set. Therefore, the selection strategy model is applicable to any IVF center in any country. In such a situation, embryologists can input their accepted twin rate setting on the model to generate their guided embryo selection strategies and present a predictable pregnancy possibility and twin rate assessment.

More important, the predictive twin risk indicated that the transferred two embryos were neither a simplified embryo implantation nor an implantation of two independent samples. This principle was previously discussed in an embryo-uterus modeling framework [32-34]. A higher twin rate than expected would be achieved if the implantation chances of the embryos transferred together were completely independent of one another [16]. Considering the variability of confounding factors, a two-level algorithm was introduced in our model for DET prediction to achieve a satisfying predictive model. Furthermore, unlike previous researches, which only considered significant influencing factors in model construction [35-37], concomitant investigation in our DET model provided a novel insight: that even if one variable were not statistically significant, it might still be important for machine learning models to predict final outcomes.

To minimize twin risk, some researchers also constructed a predictable model and validated it in subsequent IVF cycles, and the results showed a significantly reduced twin rate (from 25.2-3.8\%) [16]. However, the proportion of SETs was greatly increased (11.3-75.5\%), and surplus embryos would be preserved for future use, resulting in a reduced live birth rate in fresh cycle (29.0-25.1\%). Even though the cumulative live birth rate (CLBR) was not affected, patients had to pay more fees and spend more time for a successful pregnancy in subsequent frozen-thawed cycles. Luke et al. [38] also reported a similar conclusion of comparative CLBR and decreased multiple births in SETs over two cycles, compared to DET in one cycle. In our algorithm, conversely, the pregnancy rate and twin risk were assessed and predicted simultaneously in the fresh cycle, and further validation showed that it effectively reduced twin risk without compromising clinical pregnancy in the fresh cycle.

The recommended embryo selection strategy in our model provides decision support to embryologists with higher accuracy and efficiency. For a large proportion of IVF patients with suboptimal prognosis, which plan to choose was a dilemma to embryologists, because prediction judgment was based on their clinical experience instead of on the analysis of thousands of embryos and patient records prior to each 
embryo transfer $[39,40]$. Besides, it may also act as a counseling tool for clinicians to evaluate the chance of pregnancy before the transfer procedure.

Previous research to establish similar predictive models were problematic due to the limited number of involved features or dramatic distinction between the predicted value and the actual situation $[16,29]$. The model in the current study guides a determination of the number of embryos for transfer and the specific embryo selection, based on the analysis of a large number of features. In addition, the predicted pregnancy rate and twin risk of the corresponding selection scheme were relatively more accurate. More significantly, our model provides a flexible strategy, with individualized embryo selection for any given patient and varied operations corresponding with any setting acceptable twin rate threshold.

One shortcoming of our predictive model was that the predicted mean value was slightly elevated compared to the actual observed rate for both pregnancy and twin rates in validation, although a modest discrimination was also reported in the previous prediction model in ART [41-43]. The model should be adjusted and improved to make the predictive value more closely agree with the actual virtue in the future verification. Besides, the model was developed and validated using data from a single center. As future work, we will need to be much more rigorous with additional data sets demonstrating the predictive value, using the same parameters, as well as applying them in different centers.

\section{Conclusions}

In our study, we constructed and validated an individualized embryo selection strategy and pregnancy prediction model developed by stacking machine learning. This prediction model could provide an accurate and individualized embryo selection strategy for any given patient as well as in any twin risk threshold setting and balance a delicate correlation between clinical pregnancy and twin risk rate; it therefore promises a better IVF outcome without compromising pregnancy success and controlled twin risk.

\section{Abbreviations}

In vitro fertilization (IVF)

Single embryo transfer (SET)

Double embryo transfer (DET)

Artificial intelligence (Al)

Controlled ovarian hyperstimulation $(\mathrm{COH})$

Gonadotropin-releasing hormone $(\mathrm{GnRH})$

Human chorionic gonadotropin (hCG) 
Area under the curve (AUC)

Receiver operating characteristic (ROC)

Follicle-stimulating hormone (FSH)

Luteinizing hormone (LH)

Antral follicle count (AFC)

Logistic regression (LR)

Classification and regression tree (CART)

Assisted reproductive technology (ART)

Anti-Mullerian hormone (AMH)

Cumulative live birth rate (CLBR)

\section{Declarations}

\section{Ethics approval and consent to participate}

The study was approved by the medical ethics committee of Tongji Medical College of Huazhong University of Science and Technology and written informed consent was obtained from all patients before they were included. All methods were performed in accordance with the relevant guidelines and regulations.

\section{Consent for publication}

Not applicable.

\section{Availability of data and materials}

All data generated or analysed during this study are included in this published article.

\section{Competing interests}

The authors declare that they have no competing interests.

\section{Funding}

This work was supported by research grants from the National Key Research and Development Project (grant number: 2018YFC1002103) and the Chinese Medical Association (grant number: 16020520668) as well as the Natural Science Foundation of Hubei Province (grant number:2017CFB752). 


\section{Authors' contributions}

Q.X., M.W., B.Z., Z.L., and B.H.conducted the experiment; Q.Y., L.Y., and S.L. analyzed the data, Q.X. and Q.Y. composed the manuscript, and L.Z. and L.J. were responsible for the concept and study design. All authors contributed to the interpretation, discussion and editing of the manuscript. All authors approved the last version.

Acknowledgements Not applicable.

\section{References}

1. Sunderam S, Kissin DM, Crawford SB, Folger SG, Jamieson DJ, Warner L, et al. Assisted reproductive technology surveillance-United States, 2014. MMWR Surveill Summ. 2017;66(6):1.

2. Chambers GM, Ledger $W$. The economic implications of multiple pregnancy following ART. Semin Fetal Neonatal Med. 2014;19(4):254-61.

3. Meldrum DR, Adashi EY, Garzo VG, Gleicher N, Parinaud J, Pinborg A, et al. Prevention of in vitro fertilization twins should focus on maximizing single embryo transfer versus twins are an acceptable complication of in vitro fertilization. Fertil Steril. 2018;109(2):223-29.

4. Bergh T, Ericson A, Hillensjö T, Nygren KG, Wennerholm UB. Deliveries and children born after in-vitro fertilisation in Sweden 1982-95: a retrospective cohort study. Lancet. 1999;354(9190):1579-85.

5. Van Loendersloot L, Van Wely M, Limpens J, Bossuyt PM, Repping S, van der Veen F. Predictive factors in in vitro fertilization (IVF): A systematic review and meta-analysis. Hum Reprod Update. 2010;16(6):577-89.

6. Rienzi L, Vajta G, Ubaldi F. Predictive value of oocyte morphology in human IVF: A systematic review of the literature. Hum Reprod Update. 2010;17(1):34-45.

7. Iliodromiti S, Kelsey TW, Wu O, Anderson RA, Nelson SM. The predictive accuracy of Anti-Müllerian hormone for live birth after assisted conception: A systematic review and meta-analysis of the literature. Hum Reprod Update. 2014;20(4):560-70.

8. Fang T, Su Z, Wang L, Yuan P, Li R, Ouyang N, et al. Predictive value of age-specific FSH levels for IVF-ET outcome in women with normal ovarian function. Reprod Biol Endocrinol. 2015;13(1):63.

9. Corani G, Magli C, Giusti A, Gianaroli L, Gambardella LM. A Bayesian network model for predicting pregnancy after in vitro fertilization. Comput Biol Med. 2013;43(11):1783-92.

10. Debón A, Molina I, Cabrera S. Mathematical methodology to obtain and compare different embryo scores. Math Comput Model. 2013;57(5-6):1380-94.

11. Guerif F, Le Gouge A, Giraudeau B, Poindron J, Bidault R, Gasnier O, et al. Limited value of morphological assessment at days 1 and 2 to predict blastocyst development potential: A prospective study based on 4042 embryos. Hum Reprod. 2007;22(7):1973-81.

12. Morales DA, Bengoetxea E, Larrañaga P. Selection of human embryos for transfer by Bayesian classifiers. Comput Biol Med. 2008;38(11-12):1177-86. 
13. Roberts SA. Models for assisted conception data with embryo-specific covariates. Stat Med. 2007;26(1):156-70.

14. Zhu L, Xi Q, Zhang H, Li Y, Ai J, Jin L. Blastocyst culture and cryopreservation to optimize clinical outcomes of warming cycles. Reprod Biomed Online. 2013;27(2):154-60.

15. Zhu L, Xi Q, Nie R, Chen W, Zhang H, Li Y. Rescue intracytoplasmic sperm injection: A prospective randomized study. J Reprod Med. 2011;56(9-10):410-14.

16. Vaegter KK, Berglund L, Tilly J, Hadziosmanovic N, Brodin T, Holte J. Construction and validation of a prediction model to minimize twin rates at preserved high live birth rates after IVF. Reprod Biomed Online. 2019;38(1):22-9.

17. Chen T, Guestrin C. XGBoost: A scalable tree boosting system. Proc Int'I Conf on Knowledge Discovery and Data Mining 2016 Aug; San Francisco, CA.

18. Dunn OJ. Multiple comparisons using rank sums. Technometrics. 1964;6:214-52.

19. Benjamini $Y$, Hochberg Y. Controlling the false discovery rate: A practical and powerful approach to multiple testing. J R Stat Soc Series B Stat Methodol. 1995;57:289-300.

20. Helmerhorst FM, Perquin DA, Donker D, Keirse MJ. Perinatal outcome of singletons and twins after assisted conception: a systematic review of controlled studies. BMJ. 2004;328(7434):261.

21. Drakopoulos P, Blockeel C, Stoop D, Camus M, de Vos M, Tournaye H, et al. Conventional ovarian stimulation and single embryo transfer for IVF/ICSI. How many oocytes do we need to maximize cumulative live birth rates after utilization of all fresh and frozen embryos? Hum Reprod. 2016;31(2):370-76.

22. Van Voorhis B, Mejia RB. Single-embryo transfer point-it is the way forward. Fertil Steril. 2017;108(5):757.

23. Cai Q, Wan F, Appleby D, Hu L, Zhang H. Quality of embryos transferred and progesterone levels are the most important predictors of live birth after fresh embryo transfer: A retrospective cohort study. $J$ Assist Reprod Genet. 2014;31(2):185-94.

24. Lee TH, Chen CD, Tsai YY, Chang LJ, Ho HN, Yang YS. Embryo quality is more important for younger women whereas age is more important for older women with regard to in vitro fertilization outcome and multiple pregnancy. Fertil Steril. 2006;86(1):64-9.

25. Leijdekkers J, Eijkemans M, Van Tilborg T, Oudshoorn SC, McLernon DJ, Bhattacharya S, et al. Predicting the cumulative chance of live birth over multiple complete cycles of in vitro fertilization: An external validation study. Hum Reprod. 2018;33(9):1684-95.

26. Yanez LZ, Han J, Behr BB, Pera RAR, Camarillo DB. Human oocyte developmental potential is predicted by mechanical properties within hours after fertilization. Nat Commun. 2016;7:10809.

27. Vaegter KK, Lakic TG, Olovsson M, Berglund L, Brodin T, Holte J. Which factors are most predictive for live birth after in vitro fertilization and intracytoplasmic sperm injection (IVF/ICSI) treatments? Analysis of 100 prospectively recorded variables in 8,400 IVF/ICSI single-embryo transfers. Fertil Steril. 2017;107(3):641-48. e2. 
28. Siristatidis C, Pouliakis A, Chrelias C, Kassanos D. Artificial intelligence in IVF: a need. Syst Biol Reprod Med. 2011;57(4):179-85.

29. Minaretzis D, Harris D, Alper MM, Mortola JF, Berger MJ, Power D. Multivariate analysis of factors predictive of successful live births in in vitro fertilization (IVF) suggests strategies to improve IVF outcome. J Assist Reprod Genet. 1998;15(6):365-71.

30. Kaufmann SJ, Eastaugh JL, Snowden S, Smye SW, Sharma V. The application of neural networks in predicting the outcome of in-vitro fertilization. Hum Reprod. 1997;12:1454-7.

31. Uyar A, Bener A, Ciray H, Bahceci M. A frequency based encoding technique for transformation of categorical variables in mixed IVF dataset. Conf Proc IEEE Eng Med Biol Soc. 2009; 2009: 62146217.

32. Hunault CC, Eijkemans MJ, Pieters MH, te Velde ER, Habbema JD, Fauser BC, et al. A prediction model for selecting patients undergoing in vitro fertilization for elective single embryo transfer. Fertil Steril. 2002;77(4):725-32.

33. Roberts S, Hirst W, Brison D, Vail A, towardSET collaboration. Embryo and uterine influences on IVF outcomes: An analysis of a UK multi-centre cohort. Hum Reprod. 2010;25(11):2792 - 802.

34. Roberts SA, McGowan L, Mark Hirst W, Vail A, Rutherford A, Lieberman BA, et al. Reducing the incidence of twins from IVF treatments: Predictive modelling from a retrospective cohort. Hum Reprod. 2010;26(3):569-75.

35. Luke B, Brown MB, Wantman E, Stern JE, Baker VL, Widra E, et al. A prediction model for live birth and multiple births within the first three cycles of assisted reproductive technology. Fertil Steril. 2014;102(3):744-52.

36. Rongieres $C$, Colella $C$, Lehert P. To what extent does Anti-Mullerian hormone contribute to a better prediction of live birth after IVF? J Assist Reprod Genet. 2015;32(1):37-43.

37. Vogiatzi P, Pouliakis A. An artificial neural network for the prediction of assisted reproduction outcome. J Assist Reprod Genet. 2019;36:1441-8.

38. Luke B, Brown MB, Wantman E, Stern JE, Baker VL, Widra E, et al. Application of a validated prediction model for in vitro fertilization: Comparison of live birth rates and multiple birth rates with 1 embryo transferred over 2 cycles vs 2 embryos in 1 cycle. Am J Obstet Gynecol. 2015;212(5):676.

39. Van Der Steeg J, Steures P, Eijkemans M, Habbema JD, Bossuyt PM, Hompes PG, et al. Do clinical prediction models improve concordance of treatment decisions in reproductive medicine? BJOG. 2006;113(7):825-31.

40. Wiegerinck MA, Bongers MY, Mol BW, Heineman MJ. How concordant are the estimated rates of natural conception and in-vitro fertilization/embryo transfer success? Hum Reprod. 1999;14(3):68993.

41. Choi B, Bosch E, Lannon BM, Leveille MC, Wong WH, Leader A, et al. Personalized prediction of firstcycle in vitro fertilization success. Fertil Steril. 2013;99(7):1905-11.

42. Leushuis E, van der Steeg JW, Steures P, Bossuyt PM, Eijkemans MJ, van der Veen F, et al. Prediction models in reproductive medicine: A critical appraisal. Hum Reprod Update. 2009;15(5):537-52. 
43. Van Loendersloot L, Repping S, Bossuyt PM, van der Veen F, van Wely M. Prediction models in in vitro fertilization; where are we? A mini review. J Adv Res. 2014;5(3):295-301.

\section{Figures}

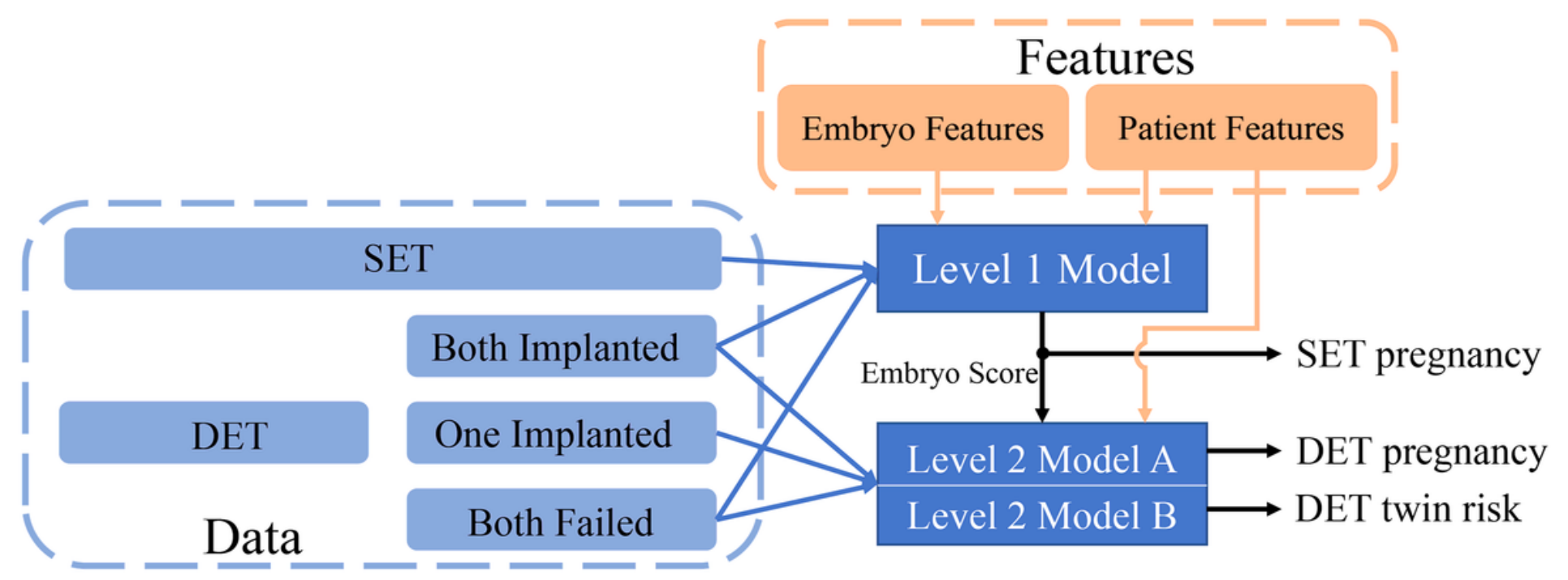

Figure 1

The overall flowchart of the proposed hierarchical model. The first-level model was trained using all data except double embryo transfer (DET), with only one embryo implantation to predict single-embryo implantation outcomes. The second level contains two models, which were trained using DET data to predict both DET implantation outcomes and twin risks. 
Predict implantation probability of each embryo

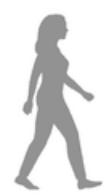

Patient

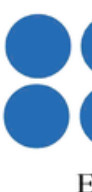

Extracted Embryos

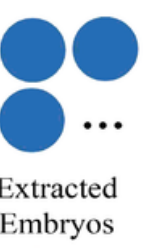

Developed plan

by our model

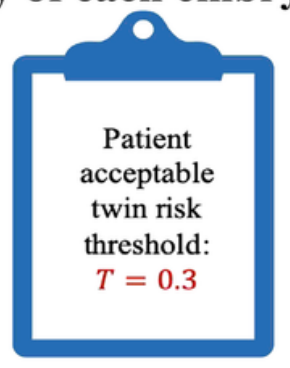

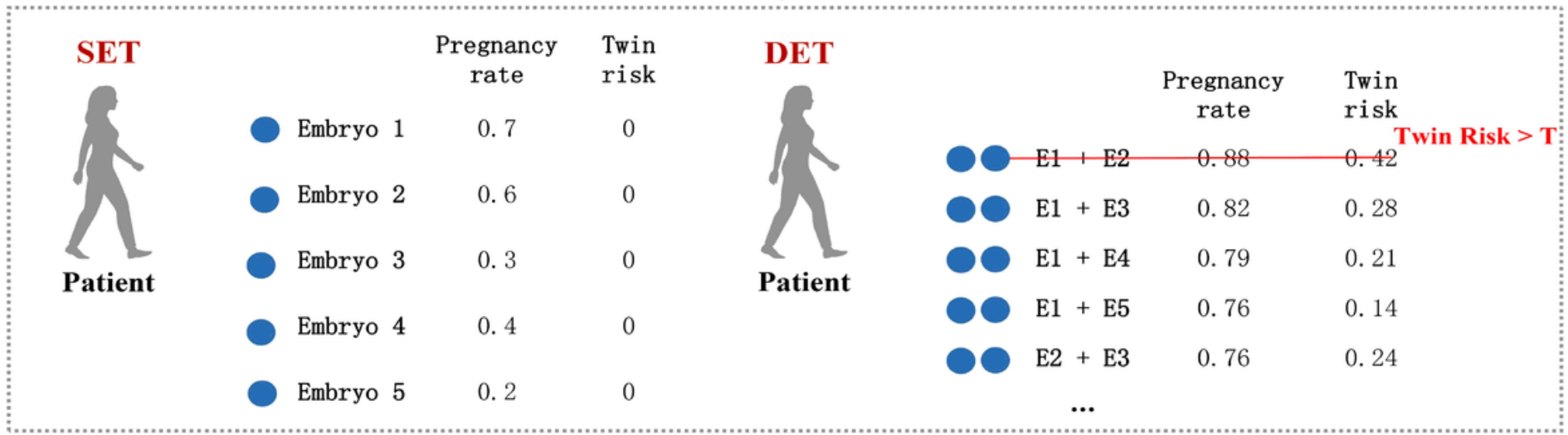

Choose the transfer plan with maximum pregnancy rate in SET/DET with controlled twin risk.

$\mathrm{E} 1+\mathrm{E} 3$

If failed, redevelop transfer plans using the embryos left.

\section{Figure 2}

Embryo selection strategy developed by the proposed model. For any given acceptable twin rate threshold, the pregnancy and twin rates could be predicted.

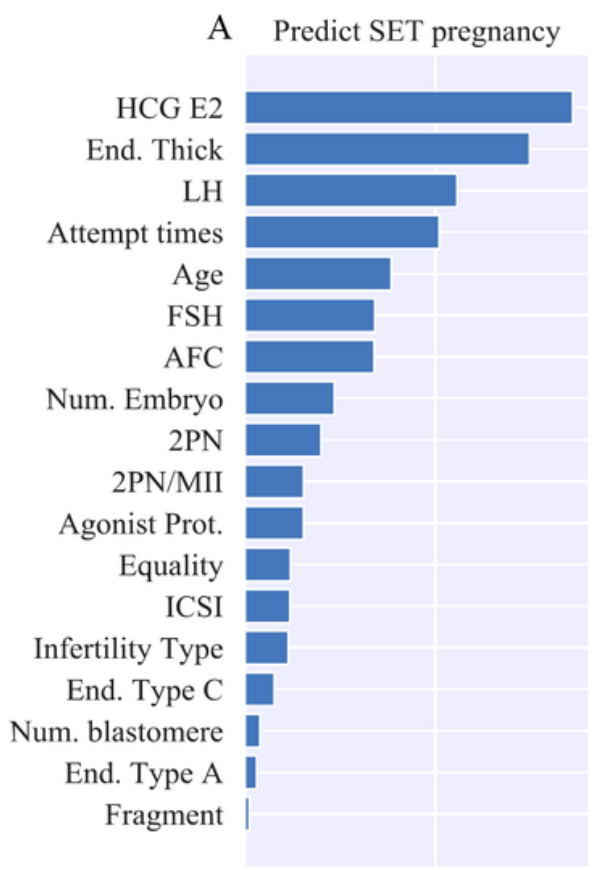

0.0
0.1
B Predict DET pregnancy

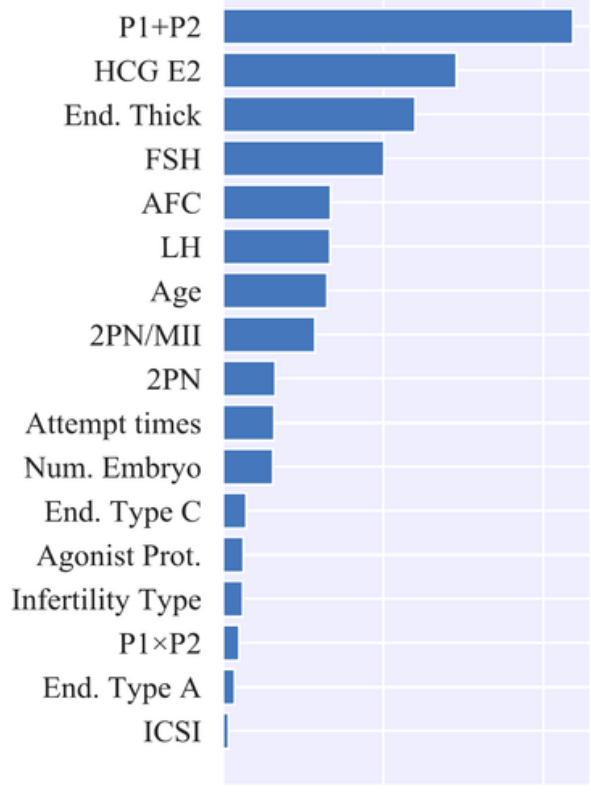

0.0
0.1

0.2
C Predict DET twin risk

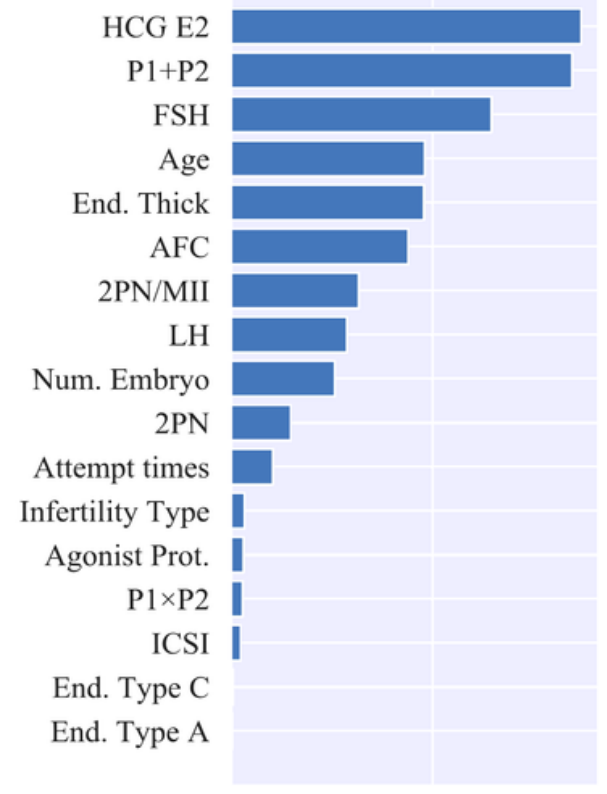

0.0

0.1 
Figure 3

Feature importance in the hierarchical model for SET pregnancy. Feature importance of (A) first-level model for predicting SET pregnancy, (B) second-level model for predicting DET pregnancy, and (C) second-level model for predicting DET twin risk.

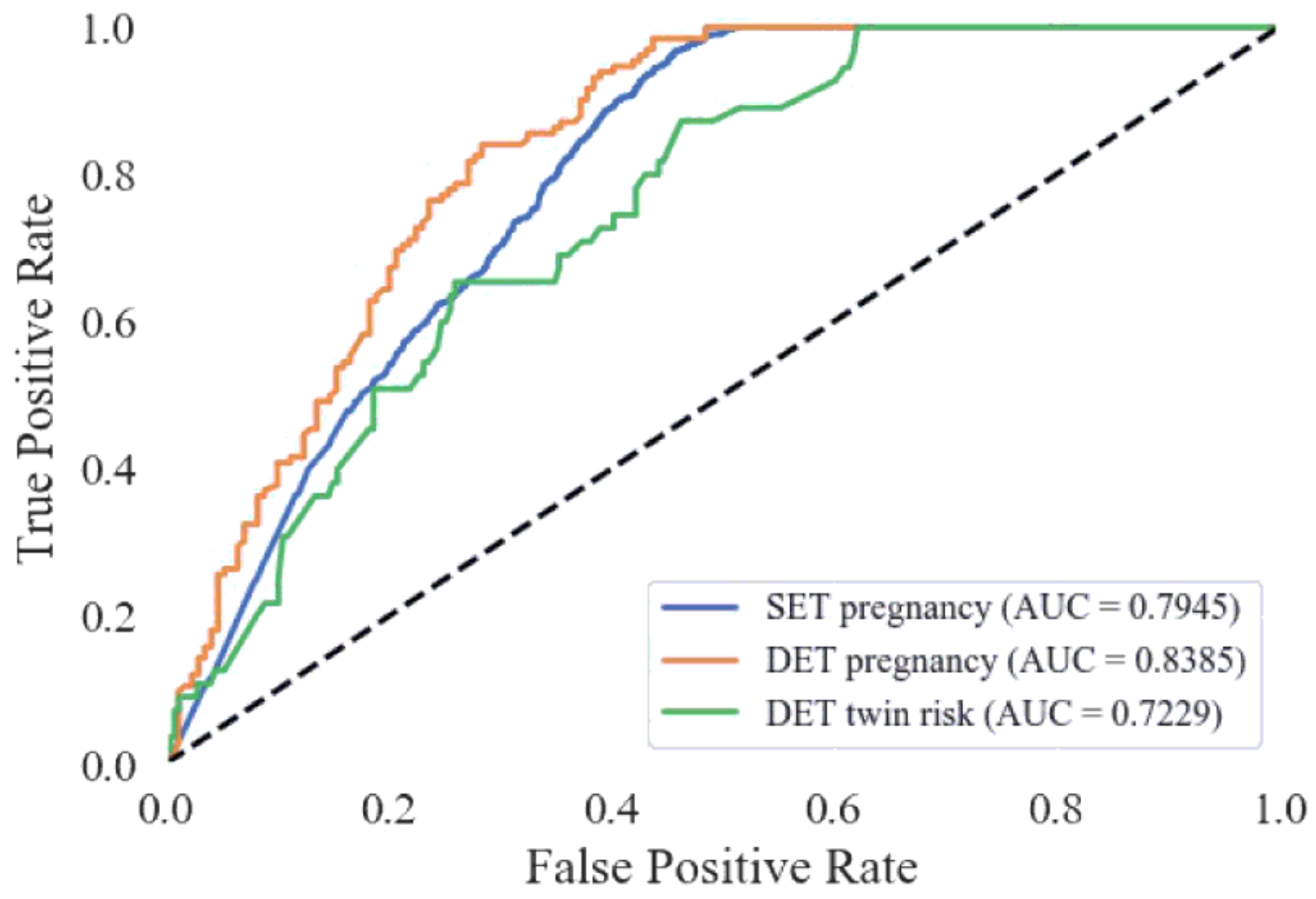

Figure 4

The ROC curve of a single-embryo transfer (SET) pregnancy, double-embryo transfer (DET) pregnancy, and DET twin risk in our method. The average AUCs for a SET pregnancy, a DET pregnancy, and DET twin risk were $0.7945,0.8385$, and 0.7229 , respectively. 

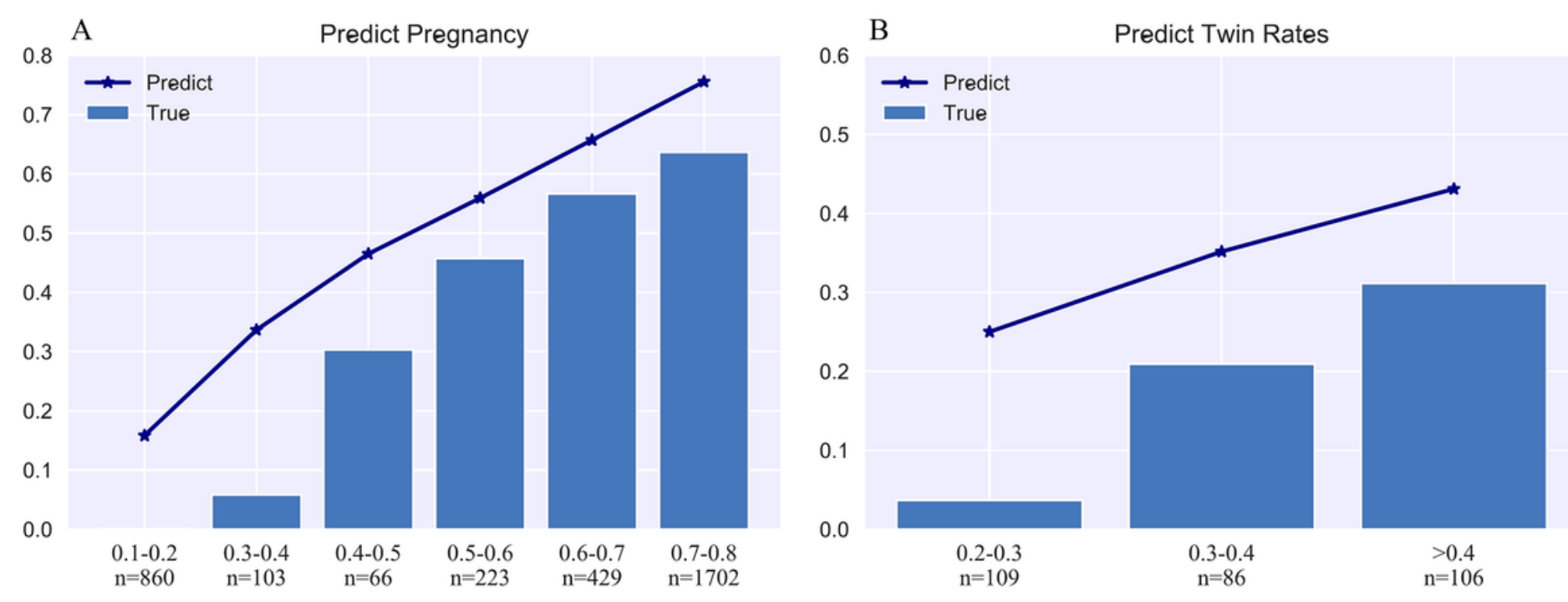

Figure 5

Validation using the predicted value with the truly observed percentage. (A) Pregnancy prediction in single-embryo transfer (SET) and double-embryo transfer (DET) versus truly observed percentage. (B) Twin risk prediction in DET versus truly observed twin rate. 
0.90

0.85

XGBoost

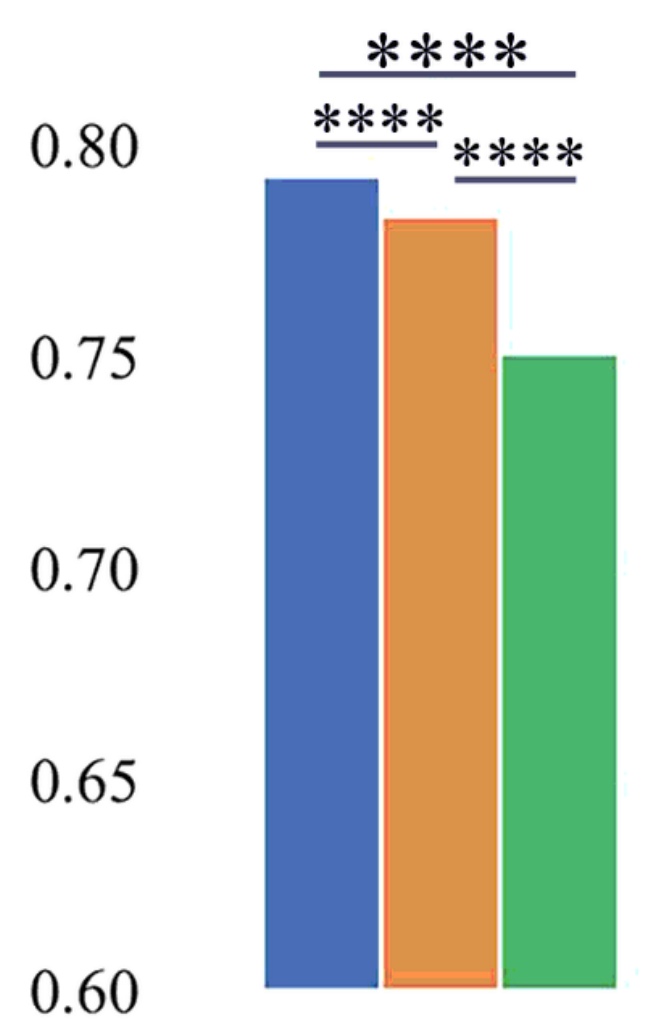

SET pregnancy

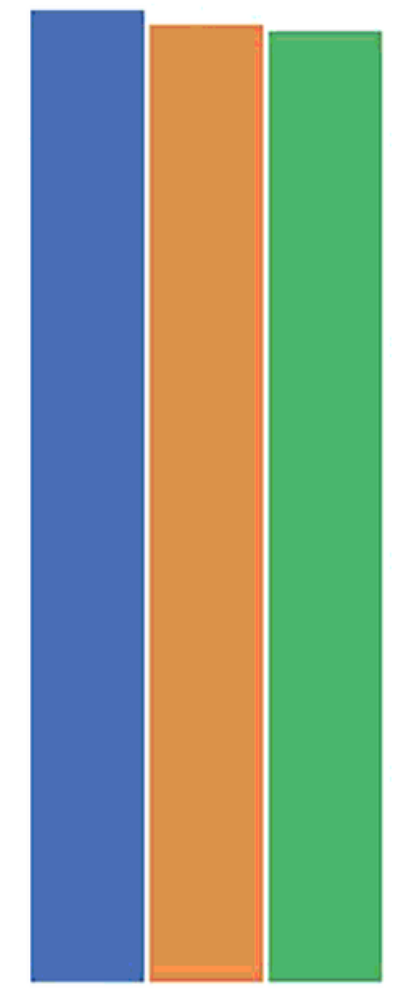

DET pregnancy

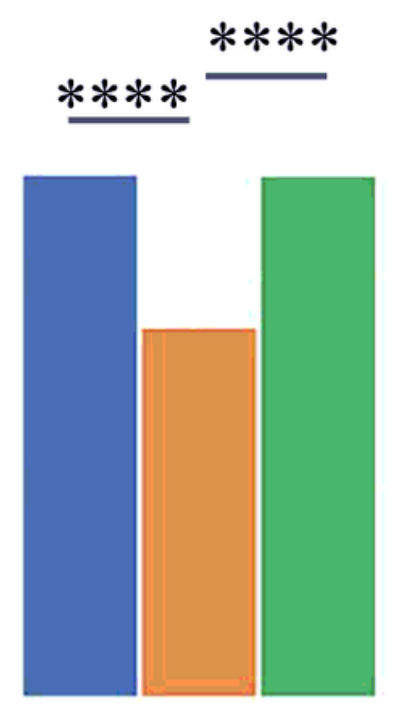

DET twin risk

Figure 6

AUC performance comparison among XGBoost, CART, and LR on single-embryo transfer (SET) pregnancy, double-embryo transfer (DET) pregnancy, and DET twin risk prediction. ${ }^{* \star \star * P} \mathrm{P}<0.0001$ 